\title{
The algebra and geometry of $\beta$
}

\author{
CHARLES E. COLLYER \\ University of Rhode Island, Kingston, Rhode Island 02881
}

\begin{abstract}
Some operations relating to signal detection theory (SDT) analysis are facilitated by expressing $\beta$ as a function of the $z$-transformed hit and false alarm rates, $z_{1}$ and $z_{0}$, respectively. This expression for the simplest equal-variance SDT model is $\beta=\exp \left[1 / 2\left(z_{0}+z_{1}\right)\left(z_{0}-z_{1}\right)\right]$.
\end{abstract}

In signal detection theory (SDT) analysis, the usual definition for and way of computing $\beta$, the observer's likelihood ratio criterion, are given by the equation

$$
\beta=\phi_{1}(\mathrm{C}) / \phi_{0}(\mathrm{C}),
$$

where $\phi_{1}(\mathrm{C})$ and $\phi_{0}(\mathrm{C})$ are the probability densities at the criterion for the signal-plus-noise and the noisealone distributions, respectively. ${ }^{1}$

The definition and computation of $\mathrm{d}^{\prime}$, the observer's discriminability index, are given by

$$
\mathrm{d}^{\prime}=\mathrm{z}_{0}-\mathrm{z}_{1}
$$

where $\mathrm{z}_{0}$ and $\mathrm{z}_{1}$ are the $\mathrm{z}$-transformed false alarm and hit rates, respectively.

Thus, the inputs to the computation of $d^{\prime}$ are $z_{0}$ and $z_{1}$, and the inputs to the computation of $\beta$ are $\phi_{0}(\mathrm{C})$ and $\phi_{1}(\mathrm{C})$; the latter are found by entering a table of the standard normal distribution with $\mathrm{z}_{0}$ and $\mathrm{z}_{1}$, respectively. If $\beta$ were expressed directly in terms of $\mathrm{z}_{0}$ and $\mathrm{z}_{1}$, then the two SDT performance measures would be algebraically and computationally more comparable. Such an expression can be derived from Equation 1 after $\phi_{0}(\mathrm{C})$ and $\phi_{1}(\mathrm{C})$ are written in their full functional forms.

The derivation is as follows:

$$
\beta=\frac{\left(2 \pi \sigma^{2}\right)^{-1 / 2} \exp \left[-1 / 2 z_{1}^{2}\right]}{\left(2 \pi \sigma^{2}\right)^{-1 / 2} \exp \left[-1 / 2 z_{0}^{2}\right]}
$$

This defines $\beta$ for the simple equal-variance yes-no case of SDT, which is the model most frequently assumed in applications. From this,

$$
\begin{aligned}
\beta & =\exp \left[1 / 2\left(\mathrm{z}_{0}{ }^{2}-\mathrm{z}_{1}{ }^{2}\right)\right] \\
& =\exp \left[1 / 2\left(\mathrm{z}_{0}+\mathrm{z}_{1}\right)\left(\mathrm{z}_{0}-\mathrm{z}_{1}\right)\right] .
\end{aligned}
$$

Portions of this paper were presented as a poster at the 1980 Eastern Psychological Association convention. I thank Norman H. Anderson, Ronald A. Kinchla, and Ewart A. C. Thomas for some of the ideas that led to this paper, and Gary Rollman for being like-minded. The author's address is Department of Psychology, Chafee Social Science Center, University of Rhode Island, Kingston, Rhode Island 02881.
With Equation 3 in hand, some useful operations are facilitated, and some interesting properties of the theory are clarified.

\section{RECOVERING HIT AND FALSE ALARM RATES}

Equations 2 and 3 constitute a pair of simultaneous equations in two unknowns, $z_{0}$ and $z_{1}$. Thus it is easy to recover unreported hit and false alarm rates from applications in which (1) $d^{\prime}$ and $\beta$ have been reported and (2) the equal-variance SDT model has been assumed. This procedure involves rewriting Equations 2 and 3 to obtain $z_{0}=\left(\ln \beta / d^{\prime}\right)+1 / 2 d^{\prime}$ and $z_{1}=\left(\ln \beta / d^{\prime}\right)-1 / 2 d^{\prime}$.

\section{THE RELATION OF $\beta$ TO d'}

Note that the expression for $\beta$ (Equation 3) contains $\left(z_{0}-z_{1}\right)$, the expression for $d^{\prime}$. This observation makes explicit the idea that $\mathrm{d}^{\prime}$ and $\beta$ are not algebraically independent. $^{2}$

One way to gain an understanding of the nature of this relation is to imagine an increase or a decrease in the separation of the noise-alone and signal-plus-noise distributions, while the criterion $\mathrm{C}$ remains fixed. The conclusion to be drawn from this thought experiment is that, in general, the probability densities at $C$ (and hence $\beta$, their ratio) will change as $d^{\prime}$ changes.

The algebraic relation of $\beta$ to $\mathrm{d}^{\prime}$ has theoretical import in the interpretation of experimental manipulations that differentially affect these measures. For example, Rollman $(1976,1977)$ has argued that some SDT applications in research on pain and analgesia have prematurely attributed changes in $\beta$ (unaccompanied by changes in $d^{\prime}$ ) to criterion shifts. He has suggested that some analgesic treatments may result in a simple shift of the sensory pain distributions to lower absolute levels, without affecting the absolute criterion for reports of severe pain. SDT is not a theory of psychophysical scaling and is not capable of discriminating such "absolute" sensory effects from criterion shifts. A constant shift of the sensory distributions without a criterion shift would have the effect of changing $\beta$ without affecting $d^{\prime}$; such a result is conventionally interpreted as a nonsensory, or "judgmental," effect of the treatment manipulation.

The algebraic form of $\beta$ confirms Rollman's (1976, 


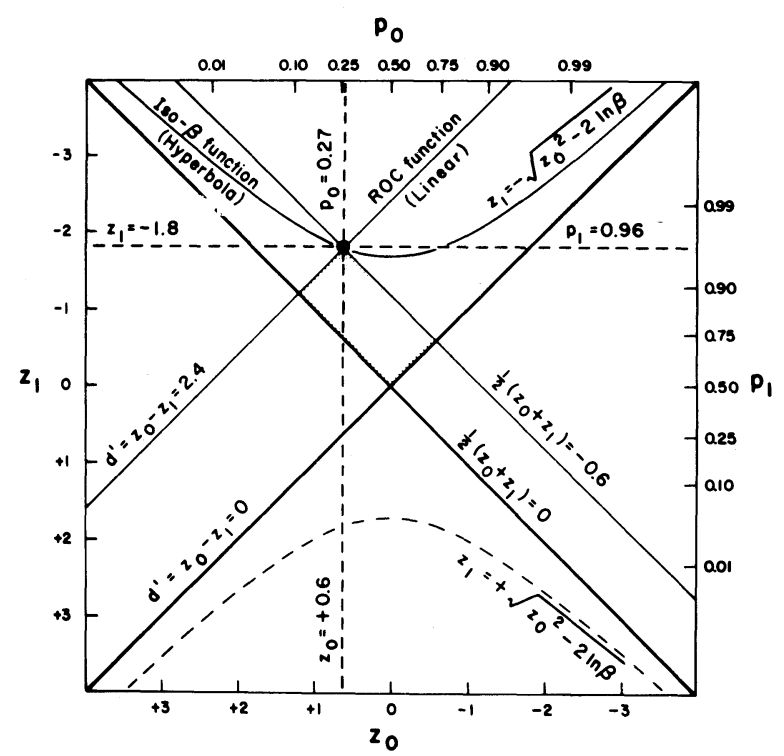

Figure 1. ROC space showing several SDT measures and functions for the performance point $z_{0}=+.6, z_{1}=-1.8$. The corresponding false alarm and hit rates are approximately $p_{0}=.27$ and $p_{1}=.96$. For this point, $d^{\prime}=2.4$ and $\beta=.24$. The area of the stippled rectangle is equal to the absolute value of $\ln \beta$.

1977) reasoning, in the following sense. Equation 3 implies that $\beta$, but not $\mathrm{d}^{\prime}$, will vary under any variation in $\left(z_{0}+z_{1}\right)$ but not $\left(z_{0}-z_{1}\right)$. This condition is met if either (1) the criterion varies but the sensory distributions do not or (2) the sensory distributions vary by addition or subtraction of a constant, but the criterion does not vary.

\section{THE GEOMETRY OF $\beta$ IN ROC SPACE}

Figure 1 represents a performance point $\left(\mathrm{z}_{0}=+.6\right.$ $\mathrm{z}_{1}=-1.8$ ) in receiver operating characteristic (ROC) space, with Gaussian scaling of the coordinate axes. I will briefly discuss some properties of this representation in order to present some interesting ways of viewing ROC space and some of the functional transformations that SDT analysis entails.

Note that if the axes of ROC space are rotated $45 \mathrm{deg}$ counterclockwise, the performance point can be represented with the new coordinates $1 / 2\left(\mathrm{z}_{0}+\mathrm{z}_{1}\right)=-.6$ and $\left(z_{0}-z_{1}\right)=+2.4$. In effect, SDT analysis transforms the representation of performance points in this way when $\mathrm{d}^{\prime}$ and $\beta$ are computed. The measure $\mathrm{d}^{\prime}$ is the ordinate value of the point after rotation, and it defines the ROC curve to which the point belongs.

The measure $\beta$ defines the abscissa value of the point after rotation, namely, the average "yes" response tendency $1 / 2\left(z_{0}+z_{1}\right)$. The performance point is the intersection of the ROC curve, the "iso- $\beta$ curve," and the new abscissa value $1 / 2\left(z_{0}+z_{1}\right)=-.6$.

Now, in analytic geometry, the canonical form of a hyperbola is $\left(\mathrm{x}^{2} / \mathrm{a}^{2}\right)-\left(\mathrm{y}^{2} / \mathrm{b}^{2}\right)=1$. Equation 3 con- forms to this general equation, with $\mathrm{a}^{2}=\mathrm{b}^{2}=2 \ln \beta$, $\mathrm{x}^{2}=\mathrm{z}_{0}{ }^{2}$, and $\mathrm{y}^{2}=\mathrm{z}_{1}{ }^{2}$. The iso- $\beta$ curve is one lobe of this hyperbola; its equation can be obtained by rewriting Equation 3 again and considering $\beta$ to be the curve parameter: $z_{1}=-\sqrt{z_{0}^{2}-2 \ln \beta}$. (The other lobe of the hyperbola lies entirely below the major diagonal of ROC space, and so it does not play any interesting role in SDT analysis.)

Finally, it is possible to visualize $\ln \beta$ in ROC space, as follows. From Equation $3, \ln \beta=1 / 2\left(z_{0}+z_{1}\right)\left(z_{0}-z_{1}\right)$. This product is the signed area of the stippled rectangle in Figure 1. Two diagonal vertices of this rectangle are the performance point itself and the origin of ROC space. The area of each such rectangle for performance points on this iso- $\beta$ curve is invariant, with a value of -1.44 .

This observation brings us back to the relationship between the SDT measures $\mathrm{d}^{\prime}$ and $\beta$. The geometry of Equation 3 suggests that $\ln \beta$ is a function of $d^{\prime}$ in the same sense that the area of a figure is a function of the length of one of its sides.

\section{CONCLUSION}

Examining the algebra and geometry of $\beta$ affords a new look at some of the functional properties of SDT. This brief treatment of these properties for the simplest case of the theory may prove useful in teaching SDT and may encourage some revision of conventional inferences drawn from SDT analysis, along the lines suggested by Rollman $(1976,1977)$ for analyses of analgesic effects.

\section{REFERENCES}

Broadbent, D. E. Decision and stress. New York: Academic Press, 1971

Rollman, G. B. Signal detection theory assessment of pain modulation: A critique. In J. J. Bonica \& D. Albe-Fessard (Eds.), Advances in pain research and therapy (Vol. 1). New York: Raven Press, 1976.

Rollman, G. B. Signal detection theory measurement of pain: A review and critique. Pain, 1977, 3, 187-211.

\section{NOTES}

1. The criterion $\mathrm{C}$ denotes a value along a univariate decision dimension that is monotonically related to likelihood ratio and may (as likelihood ratio itself may) represent a reduction of sensory evidence from an originally multidimensional raw form. Depending upon one's conceptualization of SDT, either $C$ or $\beta$ functions as a response threshold, dividing the decision dimension into lower values that map onto the "no" response and higher values that map on to the "yes" response.

2. However, the two measures are expected to be empirically independent in many experimental situations. For example, when the observer's decision rule involves "setting" $\beta$ rather than setting $C$ at some critical value, we expect $\beta$ to be determined by the a posteriori probabilities associated with sensory states, rather than by the magnitude or kind of the states themselves. See Broadbent (1971) for a discussion of the implications of these two kinds of criterion setting.

(Received for publication December 22, 1980.) 